\title{
Estudios de Grado en Cerámica en la Escuela Superior de Alcora
}

\author{
I.NEBOT-DÍAZ $(*)$, J.LLOP, M.D.NOTARI, V.MARCELO M.COLLADO \\ Escola Superior de Ceràmica de I'Alcora, L'Alcora (Castellón) \\ (*)isaac.nebot@escal.es
}

\begin{abstract}
Las enseñanzas relacionadas con la cerámica en España, cuentan con una larga tradición. Cabe destacar la importancia histórica de centros como el de Manises o la Escuela de Cerámica de la Moncloa, que a lo largo del S.XX han formado a los más ilustres ceramistas españoles. Posteriormente, otros centros han ido abriendo sus puertas para poder impartir enseñanzas relacionadas con la cerámica. En el año 2005, el último en abrir sus puertas, fue la Escola Superior de Ceràmica de l'Alcora, con el objetivo de ser el centro formador de nuevos graduados superiores para el sector cerámico.

Desde principios del S.XX, los planes de estudios relacionados con las enseñanzas puramente cerámicas, han evolucionado hasta nuestros días, hasta el punto de convertirse en Título de Grado dentro del Espacio Europeo de Educación Superior, promulgado en el Plan de Bolonia y al cual han convergido todos los estudios Universitarios.

La penúltima reforma de los Estudios Superiores de Cerámica, aprobada en el Real Decreto 2398/1998, permitió que los ceramistas comenzaran a tener una visión global de la cerámica. Esto significa, que a pesar de existir las especialidades de arte cerámico y científico-técnico, cualquier estudiante, fuese de la especialidad que fuese, tendría los conocimientos básicos de ambos itinerarios. Esta formación global, junto a la consideración de equivalencia a diplomatura universitaria, permitió que los alumnos titulados en los Estudios Superiores de Cerámica, empezaran a tener un reconocimiento en las empresas del sector.
\end{abstract}

Palabras clave: Ceramic courses, teaching, ceramic degrees

\section{Ceramic Degree Studies at L'Alcora}

Lessons related to ceramics in Spain, have a long tradition. Notably, the historic centers of Manises or school of ceramics of the Moncloa, which from the 20th century have formed the most illustrious Spanish potters. Subsequently, other centers have been opening its doors to impart lessons related to ceramics. In 2005, the last opened its doors, was the Escola Superior of Ceràmica of l'Alcora, aiming as educational centre to form new ceramic graduate in the ceramic production sector.

Since the beginning of the 20th century, related purely ceramic lessons, curricula have evolved until our days, to the point of becoming title degree within the European area of higher education adopted the Bologna plan and which have converged all university studies.

In the penultimate reform adopted by Royal Decree 2398 / 1998 the superior studies of ceramic, allowed potters began to have a global vision of ceramics. This means that in spite of the specialties of ceramic and scientific-technical art, any student be specialty was, would have the basics of both itineraries. This comprehensive training for consideration equivalent to university diploma allowed students titled the advanced ceramic studies a great recognition in companies in the sector.

Palabras clave: Cursos cerámicos, enseñanza cerámica. Titulación cerámica

\section{INTRODUCCIÓN}

Según queda recogido en el preámbulo del RD 2398/1998, de 6 de noviembre, las enseñanzas de cerámica en España cuentan con una larga tradición y un importante legado patrimonial. La relevancia de su aportación en el contexto europeo se debe, no sólo al desarrollo que este arte alcanzó en la antigüedad, sino también a la especial singularidad de la cerámica hispano-musulmana, cuya huella ejercerá una poderosa influencia en el desarrollo de las artes españolas. En la edad moderna serán las Manufacturas Reales, como la de l'Alcora fundada por el Conde de Aranda en 1727, las que impulsen de nuevo el arte de la cerámica a través de la dignificación de su oficio artesanal, al mismo tiempo que constituye el principal origen de la introducción en España de nuevos procedimientos tecnológicos y modos de organización y producción industrial, convirtiendo en la actualidad la industria cerámica en un pilar de la economía productiva nacional.

Hoy, tanto el arte como la industria comparten un interés similar por la cerámica, en cuanto a las cotas de calidad e innovación que ambos han de alcanzar para lograr anticipar soluciones óptimas a los problemas que puedan producirse en el futuro. Para avanzar en esta dirección, se ha elaborado el título de Grado en Artes Plásticas, especialidad Cerámica que garantiza la formación y adquisición de criterios para la creación y utilización de los materiales cerámicos y para el desarrollo de las capacidades artísticas y técnicas. De este 
modo, la tradicional desconexión entre creación artística y formación científica se supera a favor del beneficio que su nexo interdisciplinar confiere tanto al producto industrial como a 1 cultura y producción artística, y que tan positivamente han valorado las empresas dedicadas a la producción cerámica.

Una formación de calidad para los futuros profesionales debe contemplar un adecuado equilibrio entre los conocimientos artísticos, científicos y tecnológicos, el desarrollo de las habilidades y destrezas técnicas y procedimentales, y la capacidad para el análisis y creación de valores significativos artísticos, culturales y sociales. De este modo, el RD 634 / 2010 de 14 de mayo regula el contenido básico de las enseñanzas artísticas superiores de Grado en Artes Plásticas en la especialidad de Cerámica en su vertiente de creación artística y de formación científico-técnica, lo que garantiza que los futuros profesionales estén capacitados para actuar con eficacia, sensibilidad estética, responsabilidad, compromiso ético y respeto a los valores medioambientales e impulsar el desarrollo sostenible en los correspondientes sectores empresariales y productivos.

\section{CONTENIDOS DEL RD 634/2010.}

El RD 634/2010, de 14 de mayo, tiene por objeto establecer el contenido básico al que deben adecuarse los planes de estudio del Grado en Artes Plásticas, especialidad Cerámica, de acuerdo con los principio generales que rigen el Espacio Europeo de Educación Superior, conforme con lo regulado en la Ley Orgánica de Educación 2/2006 de 3 de mayo (LOE en adelante) y con el RD 1614/2009, de 26 de octubre, por el que se establece la ordenación de las enseñanzas artísticas superiores.

\subsection{Centros de enseñanza}

Se establece en conformidad con el artículo 58 de la LOE que estos estudios de Grado en la especialidad de Cerámica, se cursarán en las escuelas superiores de Cerámica, así como en los centros docentes privados autorizados para su impartición. En la actualidad, en España existen dos escuelas superiores de cerámica: la de Manises, y la de más reciente creación de l'Alcora (Castellón), ambas ubicadas en la Comunidad Valenciana.

Una novedad muy interesante, es la posibilidad de ofertar estudios oficiales de máster, regulados en el RD 1614/2009, al mismo tiempo que se podrán ofertar estudios de doctorado mediante convenio con las universidades.

Otra de las novedades, es la intención de convertir este tipo de centros en análogos a los centros universitarios, tal y como se conocen en la actualidad, ya que se especifica que dichos centros deben tener autonomía pedagógica, de organización y de gestión para el ejercicio de sus actividades docentes, investigadoras, creativas y de difusión de conocimiento, a fin de garantizar el cumplimiento de sus funciones como centros educativos superiores del Espacio Europeo de Educación Superior. Para ello, se fomenta el desarrollo de programas de investigación científica y técnica para poder contribuir a la generación y difusión de conocimiento y a la innovación, procurando integrarse en el Sistema Español de Ciencia y Tecnología.

\subsection{Perfiles profesionales.}

Las enseñanzas artísticas de grado en Artes Plásticas, especialidad Cerámica tendrán como objetivo la formación cualificada de profesionales que desarrollen capacidades artísticas, científicas, tecnológicas, pedagógicas, de investigación y de creación aplicadas a la innovación industrial y artística para que puedan contribuir a la mejora de la calidad de las producciones y que atiendan a los cambios sociales y tecnológicos que se vayan produciendo.

El perfil del graduado/a en Artes Plásticas, especialidad Cerámica corresponde al de un profesional cualificado capaz de entender, plantear y resolver los problemas formales, funcionales, técnicos, productivos y socioeconómicos que se puedan presentar en el ejercicio de la actividad profesional, adaptándose a la evolución de los procesos tecnológicos, industriales, concepciones estéticas y socioculturales.

\section{LA ESCOLA SUPERIOR DE CERÀMICA DE L'ALCORA.}

La Escola Superior de Ceràmica de l'Alcora (ESCAL), fue inaugurada el 15 de octubre de 2005, y su creación está aprobada en el Decreto 153/2006, de 13 de octubre del Consell de la Comunidad Valenciana.

Se trata de uno de los 13 centros pertenecientes al ISEACV -Instituto Superior de Enseñanzas Artísticas de la Comunidad Valenciana- que aglutina todos los centros de Enseñanzas Superiores de la Comunidad Valenciana, esto es 2 Escuelas Superiores de Cerámica (L'Alcora y Manises), 5 Escuelas Superiores de Arte y Diseño (Castellón, Valencia, Alcoy, Alicante y Orihuela), 3 Conservatorios Superiores de Música (Castellón, Valencia y Alicante), 2 Conservatorios Superiores de Danza (Valencia y Alicante) y 1 Escuela Superior de Arte Dramático (Valencia).

La Escola Superior de Ceràmica de l'Alcora, está ubicada en la población castellonense de l'Alcora, una de las cunas de la cerámica española y centro geográfico del entramado industrial cerámico de la provincia de Castellón, el más importante a nivel nacional y uno de los principales a nivel mundial. Su creación respondió a la demanda social y empresarial de tener un centro superior de estudios cerámicos para la formación de los profesionales de la cerámica, tanto a nivel técnico como artístico.

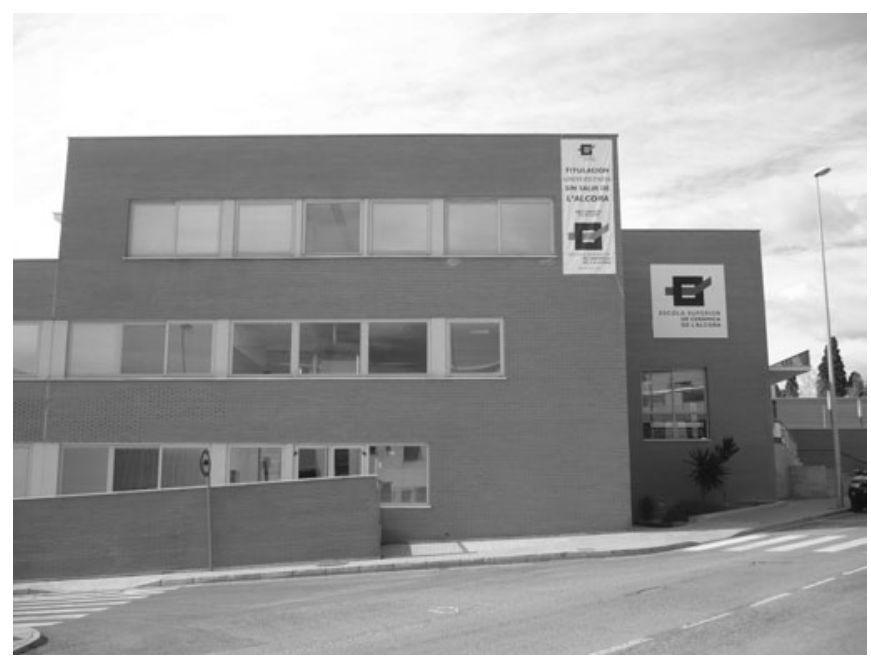


La Escola Superior de Ceràmica de l'Alcora dispone de unas amplias y completas instalaciones para la docencia de cualquier aspecto relacionado con la cerámica, bien de carácter artístico o bien de carácter técnico. Como ejemplo, destacar las salas de diseño, aulas taller individualizadas para cada una de las especialidades artísticas (volumen, modelado, decoración, ), laboratorios de investigación y desarrollo de productos, control de calidad de producto, planta piloto o sala de hornos específicos para cada tipo de producto.

Un aspecto muy importante que es conveniente destacar es la excelente relación que mantiene la Escuela con su entorno socioeconómico, y que permite un contacto directo de los estudiantes con el sector cerámico y conocer de primera mano las necesidades e inquietudes del mismo. En este sentido, la Escola Superior de Ceràmica de l'Alcora promueve todos los años diferentes actividades relacionadas con la cerámica con el propósito de acercar su actividad a la industria y diferentes colectivos sociales. Como ejemplo, cabría destacar:

- Organización de jornadas artísticas y técnicas con carácter anual.

- Participación en ferias cerámicas.

- Colaboración con empresas del sector para acercar la realidad industrial a los alumnos, mediante concursos de diseño.

- Organización y participación en cursos relacionados con la cerámica.

- Colaboración con las diferentes Asociaciones y Sociedades Cerámicas nacionales.

- Organización de exposiciones cerámicas.

\section{EL TÍTULO DE GRADO EN ARTES PLÁSTICAS, ESPE- CIALIDAD CERÁMICA.}

Tal y como se ha comentado anteriormente, las enseñanzas de grado en Artes Plásticas, especialidad Cerámica tienen como objetivo la formación cualificada de profesionales con capacidades artísticas, científicas, tecnológicas, pedagógicas, de investigación y de creación aplicadas a la innovación industrial y artística con el fin de que contribuyan a la mejora de la calidad de las producciones y atender así a los cambios sociales y tecnológicos que se vayan produciendo.

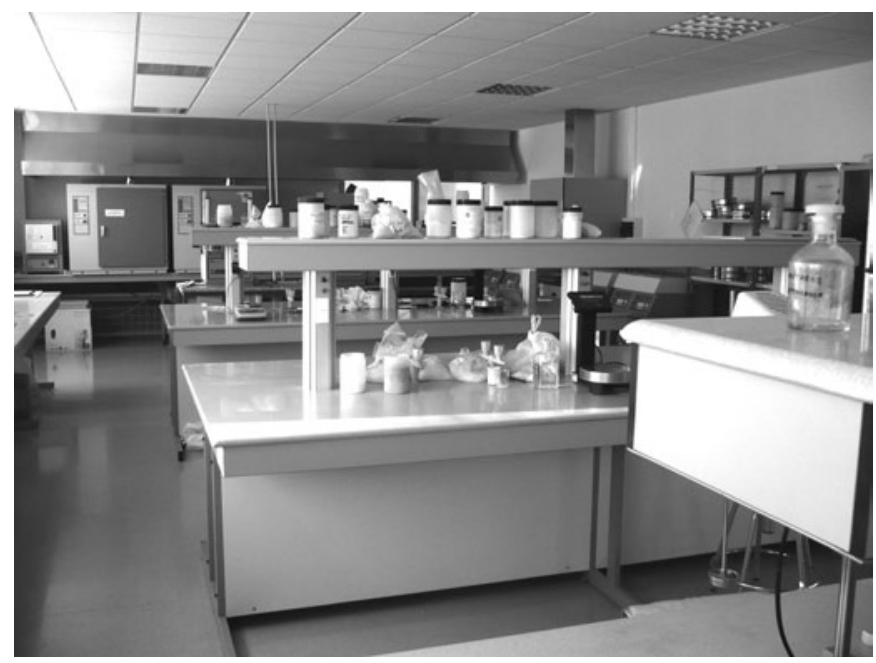

La superación de dichas enseñanzas, conduce a la obtención del Título de Graduado/a en Artes Plásticas, especialidad Cerámica que tiene carácter oficial y validez académica y profesional en todo el territorio nacional.

\subsection{Acceso}

El acceso a las enseñanzas oficiales conducentes a la obtención del Título de Graduado/a en Artes Plásticas, especialidad Cerámica, requiere estar en posesión del título de bachiller o haber superado la prueba de acceso a la universidad para mayores de 25 años.

También se abre la posibilidad de acceso para los mayores de diecinueve años de edad que no cumplan los requisitos anteriores, mediante una prueba específica que acredite que el aspirante posee la madurez en relación con los objetivos del bachillerato, así como los conocimientos necesarios para cursar con aprovechamiento las correspondientes enseñanzas.

Por último, tal y como recoge el artículo 57 de la Ley Orgánica 2/2006, de 3 de mayo, de Educación, es necesario realizar una prueba de acceso específica a estos estudios. La Resolución de 9 de Junio de 2010, de la Dirección General de Universidad y Estudios Superiores de la Conselleria de Educación de la Comunidad Valenciana, recoge en el Anexo II.2 la estructura y contenido de dicha prueba específica, y que consiste en realizar un análisis y comentario por escrito de un texto relacionado con la cerámica en el que se valora la madurez, capacidad de análisis, conocimientos asociados y el estudio del contexto histórico, científico, artístico y socioeconómico que en su caso pudiera derivarse del texto extraído.

\subsection{Plan de estudios}

Las enseñanzas de Grado en Artes Plásticas, especialidad Cerámica comprende una formación básica y una formación especializada orientada a la preparación para el ejercicio profesional. Para ello estos estudios desarrollarán, de modo integrador, capacidades artísticas, científicas y tecnológicas.

Este plan de estudios comprende 4 cursos académicos de 60 créditos cada uno, con un total de 240 créditos. Puesto que se trata de un título de grado diseñado dentro del Espacio Europeo de Educación Superior, la unidad de crédito es

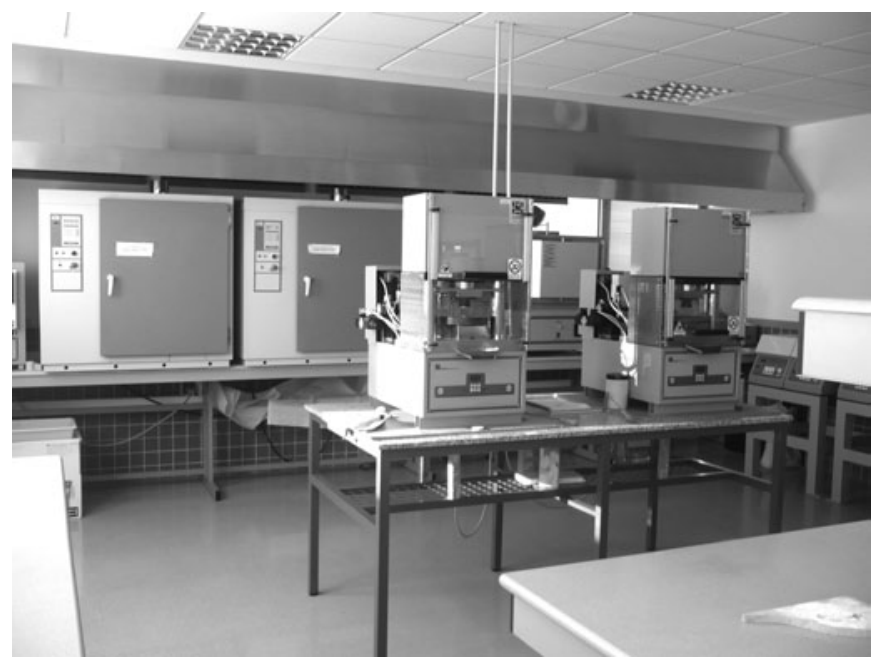


el ECTS (European Credit Transfer System), por lo que se favorece el reconocimiento y transferencia de créditos entre los diferentes sistemas universitarios europeos.

En las siguientes tablas, se muestra el resumen de todas las asignaturas que comprende el plan de estudios.

TABLA I. DISTRIBUCIÓN DE ASIGNATURAS EN PRIMER CURSO

\begin{tabular}{|l|c|}
\hline Asignatura & Créditos ECTS \\
\hline Expresión Gráfico Plástica I & 6 \\
\hline Historia y Teoría del Arte y del Diseño I & 4 \\
\hline Matemáticas & 6 \\
\hline Técnicas de Manufactura Cerámica I: Fundamentos & 4 \\
\hline Química General & 6 \\
\hline Volumen I & 4 \\
\hline Dibujo Técnico y Proyección I & 6 \\
\hline Inglés & 3 \\
\hline Laboratorio de Química General & 3 \\
\hline Materias Primas de la Cerámica & 4 \\
\hline Técnicas de Manufactura Cerámica I: Procedimientos & 4 \\
\hline Tecnología Cerámica I & 6 \\
\hline Volumen II & 4 \\
\hline
\end{tabular}

TAbla II. Distribución DE ASIGNATURAS EN SEGUNDO CURSO (IA = ITINERARIO ARTístico; ICT = ITINERARIO CiENTÍFICO-TECNOLÓGICO)

\begin{tabular}{|c|c|c|}
\hline \multicolumn{2}{|l|}{ Asignatura } & Créditos ECTS \\
\hline \multicolumn{2}{|l|}{ Dibujo Técnico y Proyección II } & 4 \\
\hline \multicolumn{2}{|l|}{ Expresión Gráfico Plástica II } & 6 \\
\hline \multicolumn{2}{|l|}{ Historia y Teoría del Arte y del Diseño II } & 4 \\
\hline \multicolumn{2}{|l|}{ Materiales Cerámicos I } & 5 \\
\hline \multicolumn{2}{|l|}{ Técnicas de Manufactura Cerámica II } & 4 \\
\hline \multicolumn{2}{|l|}{ Proyectos Básicos I } & 4 \\
\hline \multicolumn{2}{|l|}{ Optativa I } & 3 \\
\hline \multicolumn{2}{|l|}{ Ampliación de Materiales Cerámicos I } & 5 \\
\hline \multicolumn{2}{|l|}{ Proyectos Básicos II } & 4 \\
\hline \multicolumn{2}{|l|}{ Optativa II } & 3 \\
\hline Decoración Cerámica (IA) & Análisis Químico (ICT) & 6 \\
\hline Desarrollo de Proyectos Artísticos Cerámicos I (IA) & Color Cerámico (ICT) & 6 \\
\hline Laboratorio de Formas I (IA) & Tecnología Cerámica II (ICT) & 6 \\
\hline
\end{tabular}


TABLA III. DistribuCión DE ASIGNATURAS EN TERCER CURSO (IA = ITINERARIO ARTíSTICO; ICT = ITINERARIO CIENTífICO-TECNOLÓGICO)

\begin{tabular}{|c|c|c|}
\hline \multicolumn{2}{|l|}{ Asignatura } & Créditos ECTS \\
\hline \multicolumn{2}{|l|}{ Historia de la Cerámica } & 4 \\
\hline \multicolumn{2}{|l|}{ Materiales Cerámicos II } & 4 \\
\hline \multicolumn{2}{|l|}{ Moldes Cerámicos } & 8 \\
\hline \multicolumn{2}{|l|}{ Optativa III } & 6 \\
\hline \multicolumn{2}{|l|}{ Ampliación de Materiales Cerámicos II } & 4 \\
\hline \multicolumn{2}{|l|}{ Economía industrial y Estrategia de Procesos } & 4 \\
\hline \multicolumn{2}{|l|}{ Legislación aplicada al Sector Cerámico } & 2 \\
\hline \multicolumn{2}{|l|}{ Proyecto de Productos Industriales Cerámicos I } & 6 \\
\hline $\begin{array}{l}\text { Desarrollo de Proyectos Artísticos Cerámicos II: } \\
\text { Pequeño Formato }(I A)\end{array}$ & $\begin{array}{l}\text { Control y Caracterización de } \\
\text { Materiales Cerámicos I (ICT) }\end{array}$ & 4 \\
\hline Restauración Cerámica: Piezas Porosas (IA) & Análisis Instrumental I (ICT) & 4 \\
\hline $\begin{array}{l}\text { Desarrollo de Proyectos Artísticos Cerámicos II: } \\
\text { Gran Formato }(I A)\end{array}$ & $\begin{array}{l}\text { Control y Caracterización de } \\
\text { Materiales Cerámicos II (ICT) }\end{array}$ & 4 \\
\hline Laboratorio de Formas II (IA) & Tecnología Cerámica III (ICT) & 6 \\
\hline Restauración Cerámica: Piezas Vidriadas (IA) & Análisis Instrumental II (ICT) & 4 \\
\hline
\end{tabular}

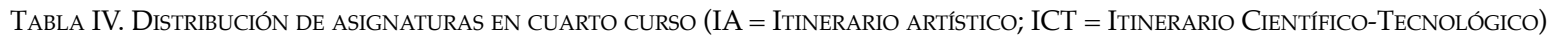

\begin{tabular}{|l|c|}
\hline \multicolumn{1}{|l|}{ Asignatura } & Créditos ECTS \\
\hline Proyecto de Productos Industriales Cerámicos II & 6 \\
\hline Optativa IV & 6 \\
\hline Optativa V & 6 \\
\hline Proyecto Fin de Carrera & 18 \\
\hline Optativa VI & 6 \\
\hline Practicum (Prácticas en Empresa) & 12 \\
\hline $\begin{array}{l}\text { Diseño Integral de Comunicación y Promoción } \\
\text { del Producto Cerámico (IA) }\end{array}$ & $\begin{array}{l}\text { Modelos Matemáticos de } \\
\text { Programación de Producción (ICT) }\end{array}$ \\
\hline
\end{tabular}


Como se puede comprobar, la formación que recibe el estudiante abarca todos los aspectos relacionados con el producto cerámico, consiguiendo una mayor profundización en un determinado aspecto dependiendo del itinerario que escoja. Además, el plan de estudios contempla un total de 6 asignaturas optativas con un total de 30 créditos ECTS, por lo que el estudiante puede diseñar un currículo acorde a sus expectativas.

\subsection{Erasmus.}

Puesto que se trata de unos estudios enmarcados dentro del Espacio Europeo de Educación Superior, también existe la posibilidad de beneficiarse de los programas Erasmus de intercambio de estudiantes. La movilidad Erasmus durante las carreras universitarias es de sobra conocida, y desde el curso 2008/2009, se abrió la posibilidad a que las Escuelas Superiores pudieran participar de estos programas, hasta la fecha únicamente válidos para Universidades.

En el artículo 18 del RD 1614/2009, se especifica que con el fin de promover la movilidad de estudiantes y titulados españoles en el Espacio Europeo de Educación Superior, junto con el título académico, se expedirá el Suplemento Europeo al Título, que es un documento que acompaña a cada uno de los títulos de educación superior de carácter oficial y validez en todo el territorio nacional, con la información unificada, personalizada para cada titulado superior, sobre los estudios cursados, los resultados obtenidos, las capacidades profesionales adquiridas y el nivel de su titulación en el sistema nacional de educación superior.

La Escola Superior de Ceràmica de l'Alcora, consiguió la Carta de Movilidad Erasmus en su modalidad Extendida, es decir, que los estudiantes del centro, además de poder realizar estancias de estudios en centros con los que se tenga el acuerdo de movilidad, puede realizar prácticas en empresas dentro de los países pertenecientes al marco de Movilidad Europea.

En la actualidad, la Escola Superior de Ceràmica de l'Alcora tiene firmados los convenios de movilidad con los siguientes centros europeos, además de estar en proceso de firma con nuevos centros:

- Cardiff Art \& Design School (Dependiente de la Universidad de Gales UWIC) con el departamento de Cerámica.

- Universidad de Bolonia, Departamento de Química Industrial.

- Istituto Superiore de la Industrie Artistiche di Faenza (Italia).

- $\quad$ Ecole Superiore de Ceramiche de Tarbes (Francia).

- HDK, University of Gotteborg (Suecia).

- AGH. Science Materials University of Krakow (Polonia).

\subsection{Máster y doctorado}

El RD 1614/2009, recoge que los centros donde se impartan las enseñanzas artísticas superiores, podrán elaborar enseñanzas de máster propios e incluso mediante convenio con universidades podrán impartir programas de doctorado.

Los estudios de máster propuestos, deben ser homologados por el Ministerio de Educación, vistos los informes del Consejo Superior de Enseñanzas Artísticas y de la agencia evaluadora correspondiente, por lo tanto son Estudios de Máster Oficiales, de validez en todo el territorio nacional.

Estos planes de estudios tendrán entre 60 y 120 créditos ECTS, que contendrán toda la información teórica y práctica que el estudiante deba recibir: materias obligatorias, materias optativas, seminarios, prácticas, trabajos, actividades de evaluación y otras que resulten necesarias según las características propias del título.

Para poder acceder a las enseñanzas oficiales de Máster será necesario estar en posesión de un Título Superior oficial de enseñanzas artísticas o un Título de Graduado o equivalente. También podrán acceder los titulados extranjeros previa comprobación por la Administración educativa competente de un nivel de formación equivalente a los requeridos.

En la actualidad, la Escola Superior de Ceràmica de l'Alcora está trabajando en la elaboración de varios títulos de Máster para poder someterlos a la homologación por parte del Ministerio de Educación.

Con todo lo expuesto, se quiere ofrecer una visión general de los estudios de Grado en Artes Plásticas, especialidad Cerámica, que se pueden cursar en la Escola Superior de Ceràmica de l'Alcora. Se trata de unos estudios de calidad para formar profesionales con un adecuado equilibrio entre los conocimientos y destrezas artísticas y científico-técnicas, y que posibilita la inserción en un sistema que requiere técnicos y artistas con un elevado nivel de formación, para así poder afrontar con firmeza y decisión el futuro del sector cerámico en este país. 